\title{
Composites polyvinyl alcohol filled with nanocellulose from oil palm waste by formic acid hydrolysis
}

\author{
Sri Aprilia ${ }^{1, *}$, Nasrullah Razali ${ }^{1}$, Yanna Syamsuddin ${ }^{1}$, Abdul Khalil, H.P.S., ${ }^{2}$ and Dedek Syafrina ${ }^{1}$ \\ ${ }^{1}$ Department of Chemical Engineering, Engineering Faculty, University of Syiah Kuala, Jln. Tgk Syech Abdur Rauf \\ no.7 Darussalam, Banda Aceh, 23111, Inonesia \\ ${ }^{2}$ School of Industrial Technology, Universiti Sains Malaysia, Penang, 11800, Malaysia
}

\begin{abstract}
Composites polyvinyl alcohol filled with nanocellulose from oil palm empty fruit bunch (OPEFB) and oil palm fronds (OPF) have investigated. Nanocellulose prepared by formic acid $50 \mathrm{wt} \%$. Functional groups and degree of crystalline of nanocellulose were analyzed by FTIR and XRD. The degree of crystalline nanocellulose presented at $2 \theta$ at intensity of 22.26 and 21.86 for OPEFB and OPF. Amorphous part of nanocellulose at intensity of 14.68 and 15.86 for OPEFB and OPF. Functional group of nanocellulose give the same pattern for OPEFB and OPF. Nanocellulose reinforce in polyvinyl alcohol as many as 1, 5, 10 and $20 \%$ and as a comparison with polyvinyl alcohol without filler. Eight of composite films and one of vinyl alcohol film have studied mechanical properties such as tensile strength and elongation at break, and also functional groups. The tensile strength of OPF 5\% and $10 \%$ increased than polyvinyl alcohol matrix. Tensile strength OPEFB $10 \%$ slightly increase from polyvinyl alcohol. The elongation of composites both OPEFB and OPF decreased with increased of nanocellulose loading.
\end{abstract}

\section{Introduction}

Oil palm waste gives attention for material composites because renewable, cheap and biodegradable materials. Oil palm solid waste such as oil palm trunks (OPT), oil palm fruit empty bunches (OPEFB), oil palm fronds (OPF), palm pressed fibers (PPF), palm kernels (PKS) and palm oil mill effluent palm (POME) are the waste that content lignin, hemicelluloses and cellulose [1]. These wastes can be produced from palm oil processing and is a problem for the environment. And if it is not handled properly will cause environmental problems for the future because almost every day it produces waste from palm oil processing. It is need technology to process oil palm solid waste to produce cellulose as reinforcement in polymer composites.

Cellulose part of oil palm waste produces after remove hemicelluloses and lignin. Cellulose is an insoluble polysaccharide that consisting of linear chains of glucopyranose units linked with a $\beta-1,4$ glycosidic bond that has a degree of polymerization of about 10,000 [2]. Cellulose is a component of plant cells that has many nanofibrils packed bundles. At nanoscale, cellulose has small number of defects to posses high modulus and tensile strength close to Kevlar [3]. The technology to produce nanocellulose from biomass has been used such as mechanical, chemical and combination methods. The common chemical procedure is acid hydrolysis. Aprilia et al. [4] insisted that acid hydrolysis of cellulose could be break of complex heterogeneous reaction to be short chains. The acid hydrolysis able to remove the amorphous part of cellulose and hemicelluloses [4]. The common acid that has been used is nitric acid $\left(\mathrm{HNO}_{3}\right)$, sulfuric acid $\left(\mathrm{H}_{2} \mathrm{SO}_{4}\right)$, hydrochloric acid $(\mathrm{HCl})$, phosphoric acid $\left(\mathrm{H}_{3} \mathrm{PO}_{4}\right)$, formic acid, etc. Several literatures state that the effect of acid concentration and time hysdrolysis gave the scale of nanocellulose size in nano range. The application of nanocellulose as reinforcement in polymer composites depend on its morphology and property [5]. The very good properties of nanocellulose such as impressive mechanical behavior, high specific surface area, good stiffeness, tensile streangth and good optical transparency [6].

The strong hydrogen bonding between cellulose crystals must be separated and good dispersion in the polymer matrices so that as reinforcing phase has good performance of nanocomposites [7]. Polyvinyl alcohol (PVA) is one of polymer plastics that can dissolve in water. PVA has high tendency to absorb water and highly hygroscopic polymer and this feature may impair its mechanical properties thus limiting its use for some applications [6].

Nanocellulose was found to be one of the interesting reinforcement to produce the friendly nanocomposites because of their nano size and the ability to chemically modify the surface and could be used in many applications. The presence of nanocellulose in polymer

*Corresponding author: sriaprilia@unsyiah.ac.id 
composites was found to improve the thermo-mechanical properties of PVA. Tan et al [8], in their review state that well dispersion and distribution of nanocellulose in PVA and excellent adhesion between nanocellulose and PVA could be improve the mechanical and barrier properties of PVA nanocomposites. The PVA nanocomposites improve the tensile modulus and tensile strength with increase nanocellulose content [7].

Although many researchers have been done to reinforce nanocellulose in PVA, there was a great variation for the degree of its reinforcement effect. In this study, OPEFB and OPF have prepared by formic acid hydrolysis to produce nanocellulose and continue to filled in PVA nanocomposites. The morphology and mechanical properties of nanocellulose and PVA nanocomposites has investigated. Morphology study for functional group and crystalline degree of nanocellulose and PVA reinforce nanocellulose. Mechanical properties has studied for PVA nanocellulose composites.

\section{Materials and methods}

\subsection{Materials and reagents}

The raw materials of OPEFB and OPF were used for nanocellulose came from smallholder plantations Nagan Raya, Kabupaten Nagan Raya, Aceh Province, Indonesia. For pulping process used $\mathrm{NaOH}$ and $\mathrm{NaOCl}$. Acid Hydrolysis process was used formic acid $50 \%$ by weight. Film nanocellulose polyvinyl alcohol was prepared PVA reinforces nanocellulose from OPEFB and OPF.

\subsection{Experimental set-up}

\subsubsection{Pulping process}

Nanocellulose isolation with acid hydrolysis by used formic acid $50 \mathrm{wt} \%$. The isolation took placed after dried and cut the OPEFB and OPF. The isolation process followed the previous method [9]. To remove lignin and hemicelluloses, approximately 20 grams of raw material was hydrolyzed using $300 \mathrm{ml}$ of $\mathrm{NaOH} 1 \mathrm{M}$ for 2 hours at $80^{\circ} \mathrm{C}$. Sample was washed by distilled water for several times by centrifuges. For bleaching process, the sample was done by used $150 \mathrm{ml}$ of $\mathrm{NaOCl} 5 \%$ for 20 minutes at temperature process at $80^{\circ} \mathrm{C}$. And then, the sample was washed with distilled water for several times. The pulp was dried in an oven at $70^{\circ} \mathrm{C}$ for 3 hours

\subsubsection{Preparation of nanocellulose}

The isolation process of nanocellulose has prepared from previous study that took formic acid 50wt $\%$ with ratio of formic acid with bleaching pulp 30:1. The process takes place in a three-neck pumpkin and stirrer at $400 \mathrm{rpm}$ and temperature $90^{\circ} \mathrm{C}$. A colloidal suspension which produced was centrifuged at $4500 \mathrm{rpm}$ for 30 minutes. After that, nanocellulose was washed with distilled water in the centrifuge for several times. The nanocellulose was dried in an oven at $70{ }^{\circ} \mathrm{C}$ for 3 hours for reinforce in PVA matrix.

\subsubsection{Preparation of film PVA nanocomposites}

Film PVA nanocomposites filled with nanocellulose from OPEFB and OPF with four loading that is $1,5,10$ and $20 \%$. We get nine film PVA nanocomposites include one film PVA without nanocellulose as a comparison.

PVA in a solution of water (10\% by weight) and a solution of nanocellulose was stirred $500 \mathrm{rpm}$ at $80{ }^{\circ} \mathrm{C}$ for 3 hours. To disperse the nanocellulose in PVA solution was used with ultrasonication for 2 minutes at $50 \%$ power level. The solution was casting on PTFE plate with thickness control $1 \mathrm{~mm}$ to form film. Plate put into a desiccator and evaporated at a temperature of $25^{\circ} \mathrm{C}$ until the film is formed. Then the film is heated in an oven at a temperature of $80{ }^{\circ} \mathrm{C}$ for 12 hours.

\subsubsection{Characterization}

The characterization of nanocellulose of OPEFB and OPF was done by FTIR and XRD. The FTIR spectra were used to identify the functional group of nanocellulose. The analyzed were recorded on Fourier Transform Infrared Spectrometer Shimadzu Prestige 6400. The amount of nanocellulose was blended with $\mathrm{KBr}$ powder and then the blending pressed into thin pellets. Nanocellulose was measured in the wavelength range from $4000 \mathrm{~cm}^{-1}$ to $400 \mathrm{~cm}^{-1}$.

Crystalline index of nanocellulose has investigated by X-Ray diffraction analyses (XRD). The measurement conditions of XRD are $40 \mathrm{kV}$ and current $30 \mathrm{~mA}$. The scattering angle range $(2 \theta)$ was from $10^{\circ}$ to $80^{\circ}$ with the rate $10 \%$ min. Eq. 1 show the formulation to determine the crystalline index (CrI) and Eq. 2 show the equation Scherer to determine size of crystalline [10].

$$
\begin{gathered}
C_{I}(\%)=\left(1-\frac{I_{a m}}{I_{200}}\right) x 100 \\
D=\frac{0.9 \lambda}{\beta_{h k l} \cos \theta_{h k l}}
\end{gathered}
$$

Crystalline index (CrI) was calculated from the maximum peak intensity at lattice diffraction (200) and Iam is the intensity minimum between the 200 and 110 peaks. And for calculating crystal size, $\mathrm{K}$ is the Scherer constant $(\mathrm{K}=0.9), \lambda$ is the wavelength of $\mathrm{X}$-ray radiation $(\lambda=1.5406 \AA)$ and $\beta 1 / 2$ is the full width at halfmaximum of the Reflection, and $\theta$ is the Bragg angle.

Mechanical test for PVA composites reinforce nanocellulos was conducted using Instron Universal Testing Machine, according to ASTM D638. The tests carry out at $10 \mathrm{~mm} / \mathrm{min}$ at $25^{\circ} \mathrm{C}$. Tensile strength and elongation at break were recorded from the software. 


\section{Results and discussion}

\subsection{Functional group nanocellulose OPF and OPEFB}

Fig. 1 show the functional group of nanocellulose OPF and OPEFB prepared from formic acid hydrolysis. Nanocellulose of OPF and OPEFB show the difference peaks at wave length $3320 \mathrm{~cm}^{-1}$. Nanocellulose OPEFB shown at peak $3320 \mathrm{~cm}^{-1}$ due to $\mathrm{OH}$ groups stretching but not clearly in nanocellulose from OPF. Shaurabh et al. [5] Investigated that at this peak was observed in this nanocellulose due to the presence of hydroxyl groups in cellulose, hemicelluloses and lignin. The peaks at wave number $2860^{\circ} \mathrm{C}$ indicated that $\mathrm{C}-\mathrm{H}$ stretching of methylene groups in cellulose. Agree with Aprilia et al, [9], at banch $1608 \mathrm{~cm}^{-1}$ indicated that this peak is $\mathrm{N}-\mathrm{H}$. At wave number $1304 \mathrm{~cm}^{-1}$ is $\mathrm{C}-\mathrm{O}$ stretching, and 1030 $\mathrm{cm}^{-1}$ shown the peaks is $\mathrm{C}-\mathrm{H}$. All of peaks in the figure have shown similarity with Aprilia et al, [4].

The three peaks at bench 1608, 1304 and $1030 \mathrm{~cm}^{-1}$ were shown for nanocellulose OPEFB and OPF. Formic acid hydrolysis show the different slightly peaks at OPF sample, but sharp at OPEFB. These peaks has came wave number at 3320 and $2860 \mathrm{~cm}^{-1}$.

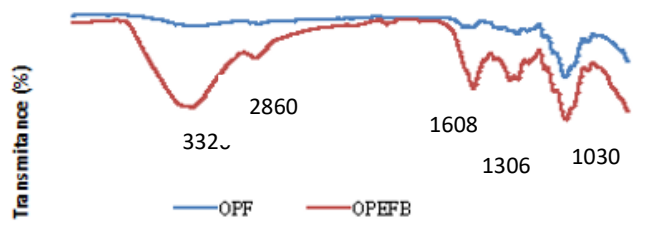

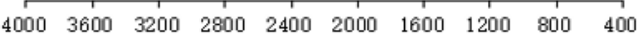
Wave number $(1 / \mathrm{cm})$

Figure 1. FTIR of nanocellulose OPF and OPEFB

\subsection{Degree of crystalline and size of nanocellulose}

Fig. 2 shown the crystalline and amorphous part of nanocellulose OPEFB and OPF that has prepared from formic acid hydrolysis. Three main replections at $2 \theta$ at Fig.2, there were came at $15.84^{\circ}, 22.50^{\circ}$ and $33.98^{\circ}$. At these peaks coresponding with the cellulose strcture Martini et al., 2014. The high intensity, both OPEFB and OPF has shown at $2 \theta=22.50^{\circ}$. This peaks investigated that the crystalline region for the sampels. The other peaks such as at $2 \theta=37.94^{\circ}, 39.42^{\circ}, 43.92^{\circ}, 57.56^{\circ}$, $64.10^{\circ}, 68.66^{\circ}$, and $77.30^{\circ}$ appear in the Fig. 2 show that the moist state content at the sample. This shows that there is still water content in the sample and the sample has not dried completely [10]. Nanocellulose prepared from OPEFB has high intensity than nanocellulose from $\mathrm{OPF}$, there are 748 and 576 for OPEFB and OPF respectively.

The size and degree of crystallinity nanocellulose from OPEFB and OPF shown in Table 1. From Table 1, the degree of crystallinity nanocellulose from OPF little bit higher nanocellulose from OPEFB. The size of crystalline of nanocellulose slightly increase nanocellulose OPF from nanocellulose OPEFB. The loose structure of cellulose cause the size to be small in the crystal size, Its cause that the degradation of smaller size as well as the defective crystal grow was responsible for increasing the size of crystal [9].

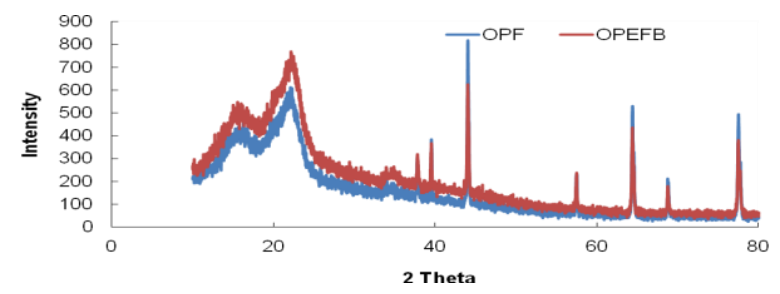

Figure 2. XRD of nanocellulose OPF and OPEFB

Table 1. Degree of crystalline and size of nanocellulose

\begin{tabular}{lcc}
$\begin{array}{c}\text { Type of } \\
\text { nanocellulose }\end{array}$ & $\begin{array}{c}\text { Degree of crystalline } \\
(\%)\end{array}$ & Size (nm) \\
\hline OPEFB & 69.60 & 38.60 \\
OPF & 70.55 & 40.45 \\
\hline
\end{tabular}

\subsection{Surface morphology of nanocellulose}

Surface morphology of nanocelluose from OPF and OPEFB are shown in Fig. 3A for nanocellulose from $\mathrm{OPF}$ and Fig. 3B for nanocellulose from OPEFB. The morphology of OPF and OPEFB are shown like rod structure. Nanocellulose OPF still show the roughnes surface but OPEFB show the smooth surface. These is impply that the OPF still have lignin and hemicellulose after hydrolysis [11]. This is also imply that at bleaching process, hemicellulose and lignin low ammount of remainning [12]. The shape as Fig. 3 indicated that nanocellulose did not bend when dried for microscopic imaging, which shows a strong crystal particle structure, due to its strong elastic properties [13]. At this result show that the structure of rod shape OPF nanocellulose image large than the OPEFB.
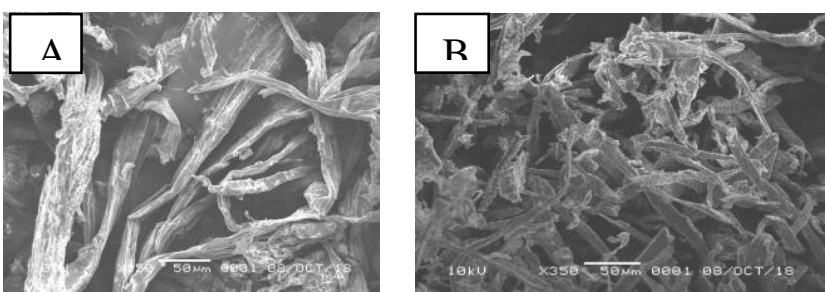

Figure 3. SEM image of nanocellulose from (A) OPF and (B) OPEFB.

\subsection{Functional group of film PVA composites}

The functional groups of film PVA composites filled with oil palm waste have investigated by FTIR. FTIR spectra of PVA film composites are shown in Fig 4 and Fig. 5. Each film PVA composites have 4 for OPEFB and 4 for OPF and also PVA without nanocellulose as comparison. All of the figures have shown the same 
pattern of wave length of PVA composites filled with nanocellulose from OPEFB and OPF. At peak $1600 \mathrm{~cm}^{-1}$ show the present of cellululose in the film composites but this peak did not appear at film PVA without nanocellulose.

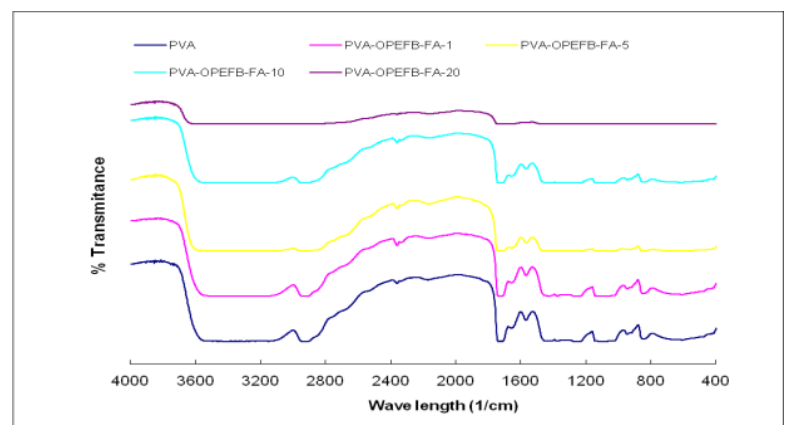

Figure 4. FTIR of PVA nanocomposites from OPEFB

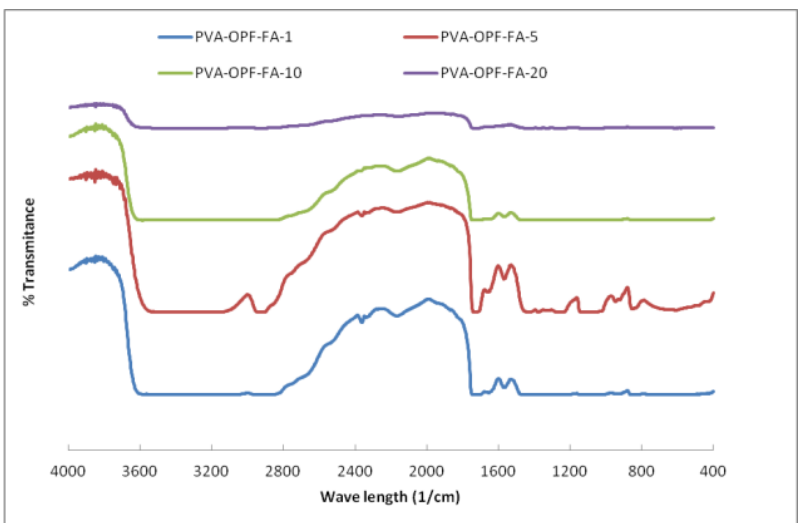

Figure 5. FTIR of PVA nanocomposites from OPF

\subsection{Mechanical analysis of film PVA composites}

Tensile strength and elongation at break of film PVA composites filled with nanocellulose from OPEFB and OPF have been investigated at Fig. 5 and 6 . There are nine film composites of PVA filled with nanocellulose from OPT and OPFB from hydrolysis formic acid and one PVA without nanocellulose as comparison. Fig. 6 show the tensile strength of composites. The PVA without nanocellulose has tensile strength 13.07 $\mathrm{kgf} / \mathrm{mm}^{2}$. The tensile strength of composites higher than PVA is PVA-OPT-20\% and PVA-OPFB-5\%, the value of tensile strength 14 and $13.07 \mathrm{kgf} / \mathrm{mm}^{2}$ respectively. The lower of tensile strength of composites are PVAOPFB-20\% and PVA-OPFB-5\%.

Tensile strength and elongation at break are improve due to the strong interaction between PVA and nanocellulose [6]. Increase nanocellulose in the matrix make the tensile strength decreased, it cause the possible aggregation of nanocellulose in the polymer matrix and also nanocellulose was not disperse homogeneously in the polymer matrix [14]. Increase in tensile strength is due to crystalline content of nanocellulose [15].

Elongation at break of film PVA composites was shown in Fig. 7. From the figure, the PVA without nanocellulose has elongation at $280.28 \%$. The higher value of elongations of composite are PVA-OPFB-1\% and PVA-OPFB-5\%, there are $358.68 \%$ and $316.16 \%$. The lowest elongation PVA composite was PVA-OPFB $20 \%$. For PVA filled with OPFB nanocellulose, elongation decrease when increasing the nanocellulo contents. But for the film composites PVA filled with nanocellulose fron OPF some increase and some decrease.

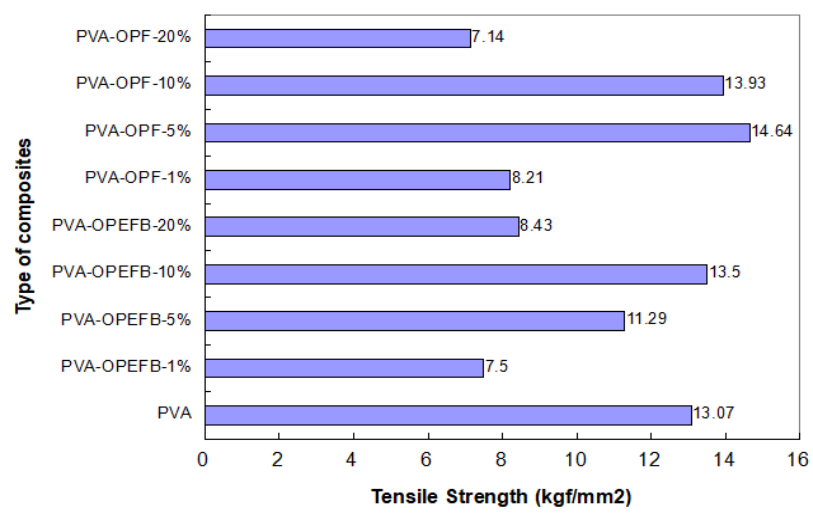

Figure 6. Tensile strength of PVA nanocomposites from OPEFB and OPF

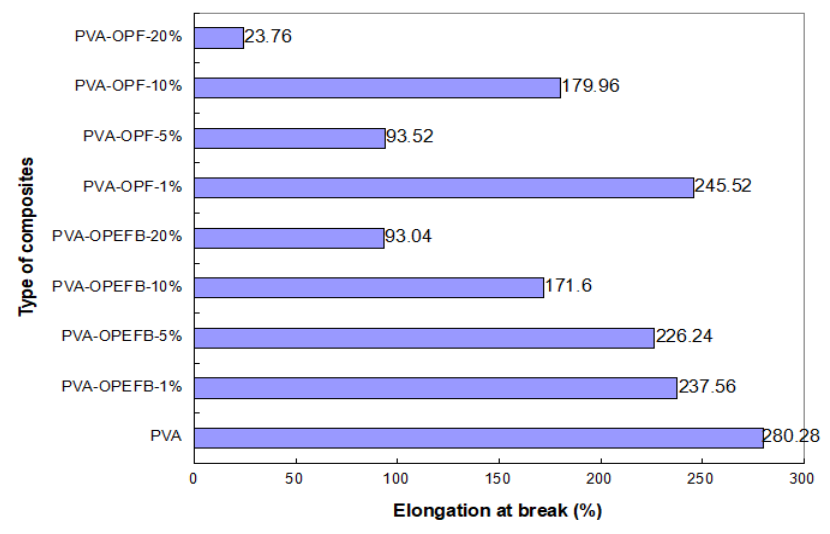

Figure 7. Elongation at break of PVA nanocomposites from OPEFB and OPF

The decreased of elongation at break of PVA composite films could be attributed to the presence of nanocellulose OPEFB and nanocellulose OPF which strain the slippage movement of PVA chain during the deformation and resulting in lower elongation at break thus decreased the chain mobilityof PVA composite films [15].

\section{Conclusion}

The goal of this work was to compare the mechanical and FTIR morphology of film PVA composites that filled with nanocellulose from OPEFB and OPF. Nanocellulose prepared by formic acid hydrolysis. FTIR result has shown that nanocellulose OPF did not appear at the peak 3320 and $2860 \mathrm{~cm}^{-1}$ of OPEFB. The average sizes of nanocellulose were about 38.60 and $40.45 \mathrm{~nm}$ for OPEFB and OPF respectively. The degree of crystalline both OPEFB and OPF closed value at 69.60 and $70.55 \%$. Morphology shows that the two samples are nano cellulose crystals in the form of rods. Both of 
nanocellulose OPEFB and OPF has good indication that the mechanical percolation (tensile strength and elongation) are affected by cellulose and matrix PVA interactions.

The authors would like to thank The Research Institutes and Community Service University of Syiah Kuala and Directorate of Research and Community Service and Directorate General of Research and Development, Ministry of Research, Technology and Higher Education who have funded this research in accordance with the Research Contract No. 59/UN11.2/PP/SP3/2018.

\section{References}

[1] S. Nasir, M. Hussein, N. Yusof, and Z. Zainal, "Oil Palm Waste-Based Precursors as a Renewable and Economical Carbon Sources for the Preparation of Reduced Graphene Oxide from Graphene Oxide," Nanomaterials, vol. 7, no. 7, p. 182, Jul. 2017.

[2] B. Chieng, S. Lee, N. Ibrahim, Y. Then, and Y. Loo, "Isolation and Characterization of Cellulose Nanocrystals from Oil Palm Mesocarp Fiber," Polymers, vol. 9, no. 12, p. 355, Aug. 2017.

[3] V. Baheti and J. Militky, "Reinforcement of wet milled jute nano/micro particles in polyvinyl alcohol films," Fibers and Polymers, vol. 14, no. 1, pp. 133137, Jan. 2013.

[4] N. A. Sri Aprilia et al., "Physicochemical Characterization of Microcrystalline Cellulose Extracted from Kenaf Bast," BioResources, vol. 11, no. 2, Mar. 2016.

[5] C. K. Saurabh et al., "Effect of Hydrolysis Treatment on Cellulose Nanowhiskers from Oil Palm (Elaeis guineesis) Fronds: Morphology, Chemical, Crystallinity, and Thermal Characteristics," BioResources, vol. 11, no. 3, Jun. 2016.

[6] A. Cataldi, D. Rigotti, V. D. H. Nguyen, and A. Pegoretti, "Polyvinyl alcohol reinforced with crystalline nanocellulose for 3D printing application," Materials Today Communications, vol. 15, pp. 236-244, Jun. 2018.

[7] Y. M. Zhou, S. Y. Fu, L. M. Zheng, and H. Y. Zhan, "Effect of nanocellulose isolation techniques on the formation of reinforced poly(vinyl alcohol) nanocomposite films," Express Polymer Letters, vol. 6, no. 10, pp. 794-804, 2012.

[8] B. Tan, Y. Ching, S. Poh, L. Abdullah, and S. Gan, "A Review of Natural Fiber Reinforced Poly(Vinyl Alcohol) Based Composites: Application and Opportunity," Polymers, vol. 7, no. 11, pp. 22052222, Nov. 2015.

[9] N. A. S. Aprilia, S. Mulyati, P. N. Alam, Karmila, and A. C. Ambarita, "Characterization nano crystalline cellulose from sugarcane baggase for reinforcement in polymer composites: Effect of formic acid concentrations," IOP Conference Series: Materials Science and Engineering, vol. 345, p. 012033, Apr. 2018.
[10] U. P. Agarwal, S. A. Ralph, C. Baez, R. S. Reiner, and S. P. Verrill, "Effect of sample moisture content on XRD-estimated cellulose crystallinity index and crystallite size," Cellulose, vol. 24, no. 5, pp. 19711984, May 2017.

[11] C. K. Saurabh et al., "Effect of Hydrolysis Treatment on Cellulose Nanowhiskers from Oil Palm (Elaeis guineesis) Fronds: Morphology, Chemical, Crystallinity, and Thermal Characteristics," BioResources, vol. 11, no. 3, Jun. 2016.

[12] N. A. Sri Aprilia et al., "Physicochemical Characterization of Microcrystalline Cellulose Extracted from Kenaf Bast," BioResources, vol. 11, no. 2, Mar. 2016.

[13] M. Ahmadi, A. Madadlou, and A. A. Sabouri, "Isolation of micro- and nano-crystalline cellulose particles and fabrication of crystalline particlesloaded whey protein cold-set gel," Food Chemistry, vol. 174, pp. 97-103, May 2015.

[14] M.-J. Cho and B.-D. Park, "Tensile and thermal properties of nanocellulose-reinforced poly(vinyl alcohol) nanocomposites," Journal of Industrial and Engineering Chemistry, vol. 17, no. 1, pp. 36-40, Jan. 2011.

[15] N. L. I. Zailuddin and S. Husseinsyah, "Tensile Properties and Morphology of Oil Palm Empty Fruit Bunch Regenerated Cellulose Biocomposite Films," Procedia Chemistry, vol. 19, pp. 366-372, 2016. 